\title{
Produktivgenossenschaften und solidarische Ökonomie als Forschungs- und Praxisfeld
}

Unter der Bezeichnung solidarische Ökonomie wird auch hierzulande seit einigen Jahren wieder kollektiv experimentiert. Es sind punktuelle Versuche, Wirtschaft und Erwerbsarbeit zu politisieren - beispielsweise in Produktivgenossenschaften jenseits etablierter Mitbestimmung. Solidarität, Demokratie, Ökologie und Gebrauchswertorientierung müssen die Akteure hier tagtäglich mit den Erfordernissen des Marktes oder öffentlicher Unterstützer vermitteln. Bisher ist die politische und akademische Resonanz eher gering, doch die Ansätze könnten zukünftig eine wichtigere Rolle spielen, sollten sich die Diskussionen um Wirtschaftsdemokratie und Alternativen zum gegenwärtigen Wirtschaftssystem intensivieren. Kritische soziologische Forschung kann dazu beitragen.

STEFAN KERBER-CLASEN

\section{Einleitung}

„Anders wirtschaften, anders leben“ - unter diesem Motto standen viele Projekte, die seit den 1970er Jahren im Zuge der Ökologie-, Frauen- und Alternativbewegungen entstanden. Eine besonders wichtige Rolle spielten dabei die selbstverwalteten oder alternativen Betriebe, die als kritische Aneignung genossenschaftlicher Traditionen verstanden werden konnten. Heute heißt es zwar auch „Eine andere Welt ist möglich“, aber Genossenschaften und andere Formen alternativen Wirtschaftens interessieren dabei kaum.

Der Nährboden der selbstverwalteten Betriebe, dieser politisch-wirtschaftlichen Experimente und der sie begleitenden intensiven politischen und wissenschaftlichen Diskurse war die Wahrnehmung vielfältiger Krisenerfahrungen: autoritäre Prozesse und Strukturen im Betrieb, patriarchale gesellschaftliche Zustände, ein bürokratischpassivierender Wohlfahrtsstaat, Wirtschaftskrise und beginnende Massenarbeitslosigkeit, ${ }^{\bullet}$ erste Warnungen vor dem ökologischen Zukunftsdesaster.

Diese Krisenphänomene haben in der einen oder anderen Form überdauert, sich teils abgeschwächt, teils verschärft, teils in ihrer Gestalt verändert; neue kamen hinzu. Ähnliches lässt sich auch für die damals gegründeten Betriebe feststellen: Einige konnten sich wirtschaftlich nicht behaupten, viele gaben bestimmte Prinzipien des alternati- ven Wirtschaftens auf und passten sich pragmatisch an die Erfordernisse des Marktes an, wenige haben die alternative Betriebsorganisation konsequent beibehalten (Heider et al. 1997; Neumann 2008).

Auch neue Formen alternativen Wirtschaftens sind in der Zwischenzeit entstanden. Das gilt nicht nur für den Dritten Sektor „gemeinnütziger bzw. zivilgesellschaftlicher Organisationen“" (Priller/Zimmer 2006, S. 17), der realwirtschaftlich von Gewicht ist und wissenschaftlich thematisiert wird, sondern auch für neue genossenschaftliche Ansätze in Form von Selbsthilfegenossenschaften Langzeitarbeitsloser (Flieger 2007a), Sozialgenossenschaften für die Erbringung sozialer Dienstleistungen (Flieger 2003) oder als Teile einer solidarischen Ökonomie (Voß 2010), die bisher faktisch marginalisiert und wissenschaftlich unterbelichtet sind. Letzteres lässt sich vor allem der Arbeits- und der Organisationssoziologie zuschreiben, die in der wissenschaftlichen Arbeitsteilung hier die Kompetenzen hätten und über analytische Werkzeuge und Begriffe verfügen, mit denen sie sich den Genossenschaften nähern könnten. Das gilt besonders für jene Forschungstraditionen, die sich

Um Arbeitsplatzverluste in einzelnen Produktionsstätten zu verhindern, wurden in den 1980er Jahren in der IG Metall Ansätze zu Betriebsübernahmen diskutiert (vgl. Bierbaum 2011). Selbstbestimmtes Wirtschaften und Arbeiten wurden dabei dem Arbeitsplatzerhalt nachgeordnet. 
als kritische Soziologie verstehen. Ohne entsprechende Hinwendung bleiben interessante Elemente einer emanzipatorischen Gestaltung von Wirtschaft und Arbeit wie bisher beinahe unerkannt und kaum diskutiert.

\section{Genossenschaften und genossen- schaftliche Forschung}

Die genossenschaftliche Landschaft in Deutschland im Allgemeinen ist gekennzeichnet durch ein Nebeneinander „liberaler" Genossenschaften und Genossenschaftsforschung einerseits und den genannten alternativen oder „sozialreformerischen" Genossenschaften ${ }^{2}$ als Teil einer solidarischen Ökonomie samt einer spezialisierten Teilöffentlichkeit andererseits.

\subsection{Liberale Genossenschaften}

In quantitativer Hinsicht sind insbesondere die liberalen Genossenschaften bedeutsam: Dietmar Berger, Vorstandsvorsitzender des „liberalen“ Fördervereins Hermann SchulzeDelitzsch, spricht bundesweit von 16,1 Mio. Mitgliedern der Kreditgenossenschaften, 2,1 Mio. Mitgliedern der verschiedenen Raiffeisen-Genossenschaften, 200.000 Mitgliedern gewerblicher Waren- und Dienstleistungsgenossenschaften, 700.000 Mitgliedern der Konsum- und Dienstleistergenossenschaften und auf Sachsen beschränkt von 590.000 Mitgliedern der Wohnungsgenossenschaften (Berger 2008, S. 413f.). Diese formalen Mitgliederzahlen geben allerdings keinen Aufschluss darüber, inwiefern bei den entsprechenden Organisationen in ihrer Wirtschaftspraxis inhaltlich von Genossenschaften gesprochen werden kann - im Sinne einer praktischen Realisierung genossenschaftlicher Prinzipien: Förder-, Identitäts-, Demokratieprinzip, Genossenschaftsgeist (Flieger 1996, S. 21ff.) - und inwiefern den Mitgliedern ihre Rolle als Genossenschafterinnen und Genossenschafter bewusst ist und sie von ihnen aktiv wahrgenommen wird.

Die Forschung an den genossenschaftlichen Instituten, die an einigen Universitäten weiterhin vertreten sind, orientiert sich thematisch an den existierenden liberalen $\mathrm{Ge}$ nossenschaften. Sie forschen in erster Linie im Sinne einer Betriebswirtschaftslehre für die bestehenden liberalen Produktivgenossenschaften und blenden so die politische Dimension weitgehend aus. ${ }^{3}$ Produktivgenossenschaften mit einer sozialreformerischen Ausrichtung, die dem Anspruch nach eine Demokratisierung des wirtschaftlichen Bereichs anstreben und zugleich Formen kollektiver sozialer Selbsthilfe sind, werden dabei nur unzureichend wissenschaftlich untersucht. ${ }^{\circledR}$ Damit geht die Historisierung der sozialreformerischen Strömung der Genossenschaftsbewegung einher, die bis zur Zerschlagung durch die Nationalsozialisten 1933 so bedeutsam wie die christlichen und liberalen genossenschaftlichen Strömungen war.
Das transportierte Selbstbild vieler liberaler Genossenschaftsmitglieder, -manager, -funktionäre, -förderer und -wissenschaftler betont sowohl die Distanz und Differenz zu rein profitorientierten Unternehmen als auch zu weltanschaulich-politischen Zielsetzungen. Leitbild wirtschaftlichen Handelns scheint sozial-verantwortliches Unternehmertum zu sein, wie es auch in Debatten um Corporate Social Responsibility diskutiert wird. Ein Beispiel soll das hier andeuten: Dietmar Berger (2008, S. 416ff.) grenzt sich ausdrücklich von den „Genossenschaftsromantikern“ und „Sozialromantikern“ ab, „die den, neuen ' Genossenschaften die Lösung aller sozialen Probleme zuordnen“, und betont als Stärke der Genossenschaften das „zuverlässige Agieren der Wirtschaftsunternehmen Genossenschaft im eigenen Territorium auf lange Sicht“.

\subsection{Sozialreformerische Produktiv- genossenschaften}

Die sozialreformerischen Genossenschaften hingegen sind bestrebt, gemeinwohlorientiert zu agieren, nicht „ausschließlich der wirtschaftlichen Besserstellung ihrer Mitglieder [zu] dienen“ (Elsen 2007, S. 276), sondern solche Ziele einzuschließen, „die weder unmittelbar noch mittelbar wirtschaftlich fördernd wirksam sind und mit einem rein ökonomistischen Verständnis des [genossenschaftlichen, SKC] Förderauftrages kollidieren“" (Flieger 2006, S. 58). Nach der verbreiteten Definition von Mersmann/Novy (1991, S. 29f.) können Genossenschaften „erst dann als sozialreformerisch gelten, wenn sie nicht bloß der Besserstellung einer Sondergruppe dienen, wenn umgekehrt also die wirtschaftlichen Vorteile der genossenschaftlichen Organisation prinzipiell verallgemeinerungsfähig sind. [...] [S] ozialreformerische Genossenschaften verstehen sich als Substitute zu Privateigentum und Marktwirtschaft (,Kooperation statt Konkurrenz', Solidarismus')“.

Verlässliche statistische Daten über die Anzahl dieser Genossenschaften, speziell der Produktivgenossenschaften, und die dort tätigen Personen gibt es nicht. Dies liegt nicht zuletzt daran, dass nicht alle die Rechtsform der eingetragenen Genossenschaft (eG) nutzen, sondern aus verschie-

(2) Im historischen Rückblick wird in der Genossenschaftsforschung unterschieden zwischen liberalen, christlichen und sozialdemokratischen bzw. sozialistischen oder sozialreformerischen genossenschaftlichen Strömungen. Die liberale Orientierung zeigt sich heute vor allem in der unhinterfragten Akzeptanz marktwirtschaftlicher Prinzipien und einer entsprechenden innerbetrieblichen Organisation.

3 Ausnahmen sind beispielsweise die Arbeiten von Kramer (2008) oder von Grosskopf et al. (2010).

(4) Einen Überblick über die aktuelle Forschung bieten die Beiträge in der einflussreichsten genossenschaftswissenschaftlichen Zeitschrift Deutschlands: der Zeitschrift für das gesamte Genossenschaftswesen. 
denen Gründen auf andere Rechtsformen zurückgreifen (z.B. e.V., GmbH). ${ }^{\oplus}$ Sicherlich existieren aktuell weitaus weniger sozialreformerische Genossenschaften als in den 1970er und 1980er Jahren.

Die dynamischste Entwicklung vollzieht sich dabei zurzeit im Feld der solidarischen Ökonomie. Seit einigen Jahren sammeln sich unter diesem Begriff, der aus der lateinamerikanischen alternativökonomischen Diskussion stammt, sowohl sozialreformerische Produktivgenossenschaften als auch andere Formen alternativen Wirtschaftens wie Tauschringe, Regionalwährungen, Konsumgenossenschaften, fairer Handel, Energiegenossenschaften, Wohnprojekte, Gemeinschaftsgärten, Umsonstläden (eine Übersicht bieten Giegold/Embshoff 2008; Müller-Plantenberg 2007; Voß 2010; Notz 2011). Es handelt sich also um eine organisatorisch, politisch und kulturell plurale Bewegung, die auf solidarische Praxis setzt und theoretische wie ideologische Grundsatzdiskussionen - etwa über den Beitrag zu einer (öko-)sozialen Transformation von Wirtschaft und Gesellschaft - in den Hintergrund stellt. Genossenschaften, verstanden als sozialreformerische Genossenschaften, kommt dabei in der wirtschaftlichen Praxis und den Diskussionen die wichtigste Rolle zu.

Gemeinsam ist den hierzulande diskutierten Begriffsbestimmungen solidarischer Ökonomie (z. B. Bernardi 2009; Giegold/Embshoff 2008; Müller-Plantenberg 2007), dass diese ein ideelles und/oder materielles Gegenmodell zu einem neoliberalen kapitalistischen Wirtschafts- und Gesellschaftssystem darstellen soll, in dem der Profitlogik, idealtypisch gesehen, andere Prinzipien des Wirtschaftens entgegengestellt werden: Orientierung an den Bedürfnissen der Menschen, Solidarität, Kooperation, Demokratie und wirtschaftliche Selbstbestimmung (Voß2010, S. 11ff.) - dies soll trotz der zumeist notwendigen Einbindung in marktförmige Prozesse versucht werden. ${ }^{\circ}$ Die genannten Prinzipien werden dabei je nach Konzept unterschiedlich gewichtet und miteinander vermittelt. An die internationale Genossenschaftsbewegung gibt es rhetorisch-programmatisch klare, aber oberflächlich bleibende Anschlüsse; eine Verankerung demokratischer Werte und Prozesse in der Wirtschaft ist als Prinzip und Ziel - ähnlich der Theorie und Praxis der Mitbestimmung und Wirtschaftsdemokratie - integraler Bestandteil der Konzepte.

Für einen Ausschnitt der solidarischen Ökonomie hat eine Projektgruppe an der Universität Kassel um die Soziologin Clarita Müller-Plantenberg ein Informationssystem erarbeitet, den Atlas der Solidarischen Ökonomie in Nordhessen. Aufgenommen wurden darin selbstverwaltete Wirtschaftsbetriebe, die zudem eine Gemeinwesenorientierung oder eine Kooperation mit ähnlichen Organisationen aufweisen und, wenn möglich, ein ökologisches Bewusstsein zeigen - denn diese "grundlegenden Charakteristika kommen nicht immer alle idealtypisch vor" (Projektgruppe Solidarische Ökonomie 2008, S. 14). Daneben wurden unterstützende, beratende und fördernde Einrichtungen miteinbezogen. Aufgezeigt werden 142 solidarische Wirt- schaftsunternehmen mit 19.003 Mitgliedern, wobei 1.092 Mitglieder und 665 Nicht-Mitglieder in den Unternehmen arbeiten, ein Großteil der Mitglieder tut dies also nicht. Ein Großteil der Unternehmen ist in der Land- und Forstwirtschaft tätig (79), wenige im verarbeitenden Gewerbe (3), in der Energie- und Wasserversorgung (4) und im Baugewerbe (2); daneben im Handel (9), Gastgewerbe (4), Grundstücks- und Wohnungswesen (11), Erziehung und Unterricht (4), Gesundheits-, Veterinär- und Sozialwesen (10) und den sonstigen Dienstleistungen. Als die wichtigsten Motive zur Genossenschaftsgründung wurden festgestellt: „Entwicklung einer Tätigkeit, bei der alle Eigentümer sind“, „Gemeinschaftliche Entwicklung von Fähigkeiten und Potenzialen“ sowie „Ergänzende Einnahmequelle für die Mitglieder“ (ebd., S. 26ff.). Ein Blick in den Atlas der Solidarischen Ökonomie in Nordhessen macht zugleich deutlich, dass viele der weniger traditionsreichen bzw. experimentellen Ansätze, die anderswo zur solidarischen Ökonomie hinzugezählt werden, ausgeklammert bleiben. Dies unterstreicht die zuvor erwähnte besondere Stellung der (Produktiv-)Genossenschaften in diesem Kontext.

\subsection{Forschungsstand zur solidarischen Ökonomie}

Die wissenschaftliche Forschung und die Zahl der Publikationen zu sozialreformerischen Genossenschaften und zur solidarischen Ökonomie sind aktuell eher überschaubar, eröffnen aber trotzdem aufschlussreiche Einblicke:

Neben dem zitierten Atlas wurden zwei Sammelbände zur solidarischen Ökonomie im Kontext von Attac organisierter Konferenzen veröffentlicht, mit Texten, die größtenteils programmatisch orientiert sind oder praktische Erfahrungen reflektieren, die in Deutschland, Italien, lateinamerikanischen Staaten oder Indien gesammelt wurden (Altvater/Sekler 2006; Giegold/Embshoff 2008). Weitere Arbeiten entstanden im Umkreis der Projektgruppe an der Universität Kassel, die die Entwicklung der solidarischen Ökonomie in Brasilien als Referenzpunkt nimmt (z.B. Müller-Plantenberg 2005, 2007). In Brasilien

5 Die Gründe dafür, genossenschaftlich außerhalb der Form der eG zu wirtschaften, sind vielfältig: Sie reichen, so die Analyse in den 1980er Jahren, von einem Informationsdefizit über diese Rechtsform über die Skepsis gegenüber der Pflichtmitgliedschaft in einem genossenschaftlichen Prüfungsverband bis zur Orientierung der Genossenschaftsverbände ausschließlich an wirtschaftlicher Konkurrenzfähigkeit (Flieger 1984). Andere Motive sind denkbar. Durch die Novellierung des Genossenschaftsgesetzes in Deutschland 2006 wurde diese Rechtsform für Genossenschaftsgründungen nach Flieger (2007b) attraktiver.

(6) Strömungen einer Umsonstökonomie verstehen sich in dieser Hinsicht als radikalerer Bruch mit einem wertvermittelten wirtschaftlichen Handeln (vgl. Habermann 2009; Uhl 2011). 
wurde, ausgehend vom Druck der Landlosenbewegung (MST) und deren Aktionen der selbstorganisierten Landbesetzung und -nutzung, von der Regierung Lula ein Staatssekretariat für solidarische Ökonomie geschaffen, welches der Sozialwissenschaftler Paul Singer seit 2003 leitet, der zugleich konzeptioneller Vordenker der brasilianischen und internationalen Diskussion ist.

Die Analyse der solidarischen Ökonomie als Ansatz zeitgemäßer sozialistischer Politik und der Blick auf die Entwicklungen in der EU stehen im Zentrum der Studien Judith Dellheims $(2005,2008)$. Diese wissenschaftlichen Arbeiten stoßen in eine wichtige Lücke, indem sie Rollen und Interessen verschiedener politischer Akteure zu bestimmen versuchen. Überfällige Geschichtsarbeit leistet ein Band mit Rückblicken auf die Alternativbetriebe (Neumann 2008), in dem der Autor zu dem Schluss gelangt, dass die „kleinen geilen Firmen“" (so der Titel des Buches) vielfach die eigenen hohen Ansprüche nicht dauerhaft erfüllen konnten. Ähnlich gelagert sind auch eine Übersicht über die Erfolge und Grenzen von Betriebsübernahmen durch Belegschaften in der Industrie von AN Maschinenbau in den frühen 1980er Jahren bis zum jüngsten Beispiel Bike Systems/Strike-Bike Nordhausen (Klemisch et al. 2010) sowie die ideengeschichtlich angelegte Auseinandersetzung Notz' mit „Theorien alternativen Wirtschaftens" (2011) und deren Einfluss auf praktische Experimente seit dem 19. Jahrhundert. Schließlich verschafft Elisabeth Voß (2010) in ihrem „Wegweiser Solidarische Ökonomie“ einen Überblick über die unzähligen Ansätze, die parallel zueinander und nicht unbedingt im Austausch miteinander im deutschsprachigen Raum existieren. Inwiefern Letzteres mit den verschiedenen Akteuren und ihren Vorstellungen des Aufbaus, der Funktionen sowie des Transformationspotenzials solidarischer Ökonomie zusammenhängt, wird in dem kompakten Überblick nicht deutlich.

Voß gehört zur Gruppe der Autorinnen und Autoren, die praktische und publizistische Erfahrungen aus der Alternativbewegung mitbringen und beide Diskurse mitgestalten bzw. -gestalteten. Gleiches gilt für Burghard Flieger, der seit den 1980er Jahren theoretische Überlegungen und arbeitssoziologische Untersuchungen veröffentlicht hat beispielsweise zu innerbetrieblicher Demokratie, Arbeitszeitgestaltung, Konflikten, Formen des Managements (z. B. Flieger 1984, 1996; Klemisch/Flieger 2007) - und dies mit genossenschaftlicher Beratung verknüpft. In seinen Arbeiten analysiert und typologisiert er verschiedene Formen von Produktivgenossenschaften und deren Umsetzung genossenschaftlicher Prinzipien in der Organisation der Arbeit und leistet zugleich die detaillierteste Auseinandersetzung mit der liberalen Genossenschaftsforschung. Er demonstriert stichhaltig deren Ignoranz gegenüber politischen Zielsetzungen von Genossenschaften und entlarvt deren prinzipielle Ablehnung von Produktivgenossenschaften als ideologisch.

Feministische Perspektiven bringen die Autorinnen Friederike Habermann (2009), Sibylle Plogstedt (2006) und
Gisela Notz $(1991,2004,2011)$ in die Debatten ein. Plogstedt untersucht den Weg der selbstverwalteten Frauenbetriebe von ihrem Beginn bis zur Gegenwart und konstatiert dabei einen Trend des Übergangs vom Kollektivbetrieb zu kooperierenden Einzelunternehmerinnen. Habermann unternimmt ähnlich wie Voß den Versuch, die Breite der Ansätze alternativen Wirtschaftens zu erfassen. Mit dem Bild der Halbinsel verdeutlicht sie dabei den Status dieser Projekte - nicht als isolierte Inseln im Meer, sondern immer verkoppelt mit der umgebenden Gesellschaft und Wirtschaft und so prinzipiell auch miteinander müssten sie gedacht werden. Das bedeutet zugleich, sie nicht nur kapitalistischen Strukturen gegenüberzustellen, sondern auch patriarchalen und rassistischen Ungleichheitsstrukturen. Notz schließlich setzt sich seit dem Beginn der Alternativ- und Frauenbewegung für feministische Analysen und Positionen ein und macht zugleich deutlich, dass diese, trotz der Beteiligung vieler Frauen in diesen Betrieben, bisher stets wissenschaftlich und publizistisch marginal geblieben sind. Lediglich einige Arbeiten in den 1980er Jahren diskutierten die nach wie vor wichtigen Fragen der Anzahl der Frauen in diesen Genossenschaften, die geschlechtsspezifische Arbeitsteilung, Löhne und Arbeitszeiten, Vereinbarung von Sorgeund Erwerbsarbeit und informelle Hierarchien aus der Perspektive feministischer Arbeitssoziologie.

Aus einer gewerkschaftlichen Perspektive argumentieren Heinz Bierbaum und Marlon Riege (1989), die in den 1980er Jahren gemeinsam versuchten, genossenschaftliche und gewerkschaftliche Diskussionen in einen praktisch und theoretisch angelegten Dialog miteinander zu bringen. Diesen Versuch aktualisierten Irmtraud Schlosser und Bodo Zeuner (2006). Wichtig war dabei stets die Frage nach der Selbstausbeutung - der „freiwilligen“ Akzeptanz geringerer Löhne, längerer Arbeitszeiten und geringerer sozialer Absicherung. Dieses Problem stellt sich heute, angesichts zunehmender Prekarisierung, womöglich anders. Kürzlich nahm Bierbaum (2008) diesen Faden wieder auf, um Genossenschaften als praktisches Gegenmodell zu finanzmarktgetriebener Unternehmensführung in die Diskussion zu bringen - anerkennend, dass sich mit dem Leitbild der Ich-AG auch auf diskursiver Ebene die Kräfteverhältnisse im Vergleich zu den 1980er Jahren deutlich zulasten kollektiver Wirtschaftsformen verschoben haben. Wobei die aktuelle Krise diese neoliberale Deutungshoheit praktisch untergraben habe (Bierbaum 2011). Schließlich spielt die Zeitschrift Contraste eine nicht zu unterschätzende Rolle als Sprachrohr und Diskussionsforum der Alternativbewegung und der Akteurinnen und Akteure der solidarischen Ökonomie, mithin als eine Form der Gegenöffentlichkeit. ${ }^{\circledR}$ Hier werden betriebliche Erfahrungen genauso reflektiert und diskutiert wie übergreifende Fragen sozialer Bewegungen.

(7) Contraste: Monatszeitung für Selbstorganisation, http:// www.contraste.org. 
Mit Ausnahme einiger Arbeiten von Flieger, Notz und der Arbeit von Plogstedt vereint alle diese aktuellen Texte, dass es sich hierbei nicht um theoretische oder empirische arbeitssoziologische Studien handelt: Es sind insbesondere politisch-programmatische Reflexionen, Überblicke über die unterschiedlichen praktischen Ansätze, historische Analysen oder essayistische Texte, welche die Möglichkeiten alternativen Wirtschaftens allgemein oder mit Blick auf nationale Entwicklungen eher optimistisch erörtern. Aus dieser Tatsache folgt, dass soziologische Erkenntnisse zur Organisation des Arbeitsprozesses, zur innerbetrieblichen Demokratie, zur Motivation der Genossenschafterinnen und Genossenschafter, zur Entlohnung, zu den Arbeitsbedingungen, zur geschlechtlichen Arbeitsteilung und zu vielen weiteren potenziell interessanten Aspekten sozialreformerischen produktivgenossenschaftlichen Wirtschaftens aktuell weitgehend fehlen. Dies bedeutet aber auch, dass Ansätze, Konzepte und Analysen aktueller arbeitssoziologischer Forschung nicht an diesem Forschungsgegenstand geprüft, geschärft und überdacht werden können: z. B. Subjektivierung und Entgrenzung von Arbeit, innerbetriebliche Partizipation, Gestaltung von Entgeltsystemen, Lernen am Arbeitsplatz, Beschäftigungssicherung, Vermittlung von Arbeit und Leben.

\section{Genossenschaften und Wirtschaftsdemokratie}

In öffentlichen und politischen Debatten kommen Produktivgenossenschaften von Zeit zu Zeit am Rande vor. Ihnen werden verschiedene Funktionen und wirtschaftliche Tätigkeitsbereiche zugeschrieben: als Wirtschaftsform, die weniger unter der wirtschaftlichen Krise leidet und die Standortverlagerungen weder androht noch vollzieht, als Möglichkeit zur Schaffung von Arbeitsplätzen für am Arbeitsmarkt benachteiligte Menschen oder als Lückenbüßer ehemals sozialstaatlicher Leistungen. Bei manchen Vertreterinnen und Vertretern eher gesellschaftskritischer Positionen werden sie thematisiert als Form der kollektiven Selbsthilfe Arbeitsloser (Wir-eG), als Alternative zur Privatisierung kommunalen Eigentums, als innovativer Ansatz bürgerschaftlicher Teilhabe, als Form des Wirtschaftens nach alternativen Werten oder als Element wirtschaftsdemokratischer Konzepte und Utopien. Bei genauerem Hinsehen zeigt sich, dass Genossenschaften hier zumeist als Stichwort wieder eingeführt werden, ohne dass eine Auseinandersetzung mit genossenschaftswissenschaftlichen Begriffen und historischen Praxen stattfindet. Dies zeigt sich beispielsweise an der Ignoranz gegenüber verschiedenen Versuchen, genossenschaftliche Prinzipien für die heutige Wirtschaft und Gesellschaft zu definieren, um diese Wirtschaftsform von anderen abzugrenzen, oder in der ungenauen Bezeichnung des Gegenstandes, bei der Genossen- schaft gleichbedeutend mit Produktivgenossenschaft verwendet wird oder verschiedene Genossenschaftsformen (Produktiv-, Konsum-, Wohnungsbaugenossenschaften etc.) und -typen (Förder-, Produktiv- und Vollgenossenschaften) begrifflich zusammenfallen.

Ähnliche Probleme zeigen sich in der Rezeption genossenschaftlicher Konzepte und Analysen. Dies soll beispielhaft verdeutlicht werden an der Diskussion um Wirtschaftsdemokratie, die in jüngster Zeit wieder intensiver geführt wird.

Konzepte der Wirtschaftsdemokratie stehen in der deutschen Diskussion in der gewerkschaftlichen Tradition Fritz Naphtalis. Unter anderem darin liegt begründet, dass von den beiden ursprünglichen Bewegungen der Demokratisierung der Wirtschaft, die Mitte des 19. Jahrhunderts ihren Ausgangspunkt nehmen - Gewerkschaftsbewegung und Genossenschaftsbewegung - traditionell Erstere vorrangig thematisiert wird. Konzepte der Wirtschaftsdemokratie schließen also in erster Linie an die konkreten demokratischen Elemente, Erfahrungen und Akteure der Mitbestimmung an (Demirović 2007) und versuchen auf den verschiedenen Ebenen eine erweiterte und vertiefte Demokratisierung $\mathrm{zu}$ theoretisieren und voranzubringen (z. B. Vilmar 1999; Krätke 2003, 2008; Demirović 2007, 2011; Beerhorst 2009; Martens 2010). Produktivgenossenschaften spielen dann teilweise überhaupt keine Rolle, wie etwa bei Beerhorst, oder werden nur in verkürzter Weise integriert.

Vilmar (1999, S. 20) führt Produktivgenossenschaften als „älteste Struktur der Wirtschaftsdemokratie“ in sein Konzept von Wirtschaftsdemokratie rhetorisch ein, argumentiert aber zugleich, dass diese durch eine Kombination von verschärfter Gewerbeaufsicht und erweiterter Mitbestimmung auch ersetzt werden können. Krätke hingegen räumt Produktivgenossenschaften einen zentralen Platz in seinem Konzept ein. Wenn er die gesamte Bewegung der Genossenschaften als Eckpfeiler einer demokratischen Wirtschaftsordnung und potenzielle Basis eines sozialistischen Transformationsprozesses charakterisiert (Krätke 2008, S. 102f.), abstrahiert er allerdings zu sehr von den Zielen und dem jeweiligen Selbstverständnis der Mehrheit der real existierenden Genossenschaften.

Beide Auffassungen werden weder den spezifischen Prinzipien und Prozessen produktivgenossenschaftlichen Wirtschaftens gerecht noch einer genaueren Analyse der derzeitigen genossenschaftlichen Unternehmen und Akteure: Würden sich Befürworter eines sozialistischen Transformationsprozesses vor allem auf traditionell konservative Agrargenossenschaften stützen (wollen)?

\section{Ansatzpunkte kritischer Forschung}

Eine weitergehende arbeitssoziologische Forschung könnte an verschiedene Aspekte der skizzierten Genossen- 
schaftsforschung und -praxis anschließen: Verstanden als kritische Wissenschaft, könnte die Perspektive einer weitergehenden Demokratisierung der Wirtschaft das Leitmotiv genossenschaftlicher Forschung darstellen. Damit würden insbesondere sozialreformerische Produktivgenossenschaften in den Fokus der Aufmerksamkeit rücken.

Eine Arbeitssoziologie dieser Genossenschaften würde dabei nicht bei null anfangen müssen, denn die reichhaltige Forschung zu den alternativen Betrieben der 1970er und 1980er Jahre sowie vereinzelte spätere Studien (z. B. Flieger 1996; Plogstedt 2006; Klemisch et al. 2010) bieten Anknüpfungspunkte, die erschlossen werden können. Diese ermöglichen es, aktuelle Forschungsperspektiven nicht nur auf kapitalistische und öffentliche Unternehmen, sondern auch auf Genossenschaften zu richten. Anknüpfungspunkte bietet auch die internationale Forschung: In Frankreich, Italien, Spanien, Brasilien oder den USA existieren Forschungstraditionen, die sozialreformerische Aspekte von Genossenschaften thematisieren, wenngleich die Rezeption dieser Arbeiten durch die Verwendung der jeweiligen Landessprachen teilweise erschwert wird. Auch dürfen die nationalen Besonderheiten der Rahmenbedingungen und Traditionen genossenschaftlichen Wirtschaftens hierbei nicht vernachlässigt werden: Das sich im Zeitverlauf entwickelnde und wandelnde Selbstverständnis von Genossenschaften und Genossenschaftsbewegungen sowie die Kooperation mit anderen politischen Akteuren (z. B. Gewerkschaften) müssten dabei genauso Beachtung finden wie die Wirtschaftsbereiche, in denen Genossenschaften aktiv sind; öffentliche Interventionen in die Wirtschaft genauso wie wohlfahrtsstaatliche Politiken, die kollektive Selbsthilfe in unterschiedlichem Maße notwendig machen.

Kritische theoretische Überlegungen zu genossenschaftlichem Wirtschaften sind beispielsweise im englischsprachigen Diskurs präsent und könnten einen hierzulande im Moment eher theoriearm geführten Diskurs bereichern. So gibt es dort Versuche, Produktivgenossenschaften aus (neo-)marxistischen Perspektiven zu theoretisieren. Im Anschluss an Marx und Gramsci identifizieren etwa Atzeni/ Ghigliani (2007), Baldacchino (1990a, 1990b), Egan (1990) und Jossa (2005, 2009a, 2009b) Probleme, Potenziale und Rahmenbedingungen von Produktivgenossenschaften: Die Betonung der Bedeutung progressiver Bündnisse als wichtige Rahmenbedingung, um die Ziele genossenschaftlichen Wirtschaftens zu erreichen, kann dabei als einer der immer wiederkehrenden Aspekte (neo-)marxistischer Genossenschaftstheorie festgehalten werden. Schon Marx äußerte sich in dieser Hinsicht zur Notwendigkeit der Bildung von materiellen und immateriellen genossenschaftlichen Verbünden, um gegenseitige Konkurrenz zu vermeiden und die Gründung neuer Genossenschaften finanziell zu unterstützen (Marx 1973, S. 28; Jones 1979, S. 579f). ${ }^{\circledR}$ Rosa Luxemburg (1899), die Genossenschaften skeptisch gegenüberstand, führte als weiteres Element die bewusste Schaffung von Absatzmärkten in Kooperation von Produktivgenossenschaften und Konsumgenossenschaften in die- sen Diskurs ein. Schließlich ist es die Kooperation mit sozialdemokratischen und sozialistischen Parteien und Gewerkschaften, der in dieser Perspektive das Potenzial zugesprochen wird, die politischen und ökonomischen Rahmenbedingungen genossenschaftlichen Wirtschaftens zu verbessern.

Dabei werden die produktivgenossenschaftlichen Ziele darin gesehen, praktische antikapitalistische Politik zu betreiben, Wirtschaftsdemokratie zu verbreiten und zu vertiefen und assoziierte Produktion als sozialistische Produktionsweise vorwegzunehmen - zur Verbesserung der gegenwärtigen Arbeits- und Lebensbedingungen, zur Vorbereitung revolutionärer Praxis oder um einen eigenständigen genossenschaftlichen Sektor zu etablieren. Damit soll zugleich die politische Demokratie ergänzt und abgesichert und der gesellschaftliche Reichtum gerechter verteilt werden.

Dies geschieht in dieser Sicht allerdings in einem System der Klassenherrschaft, das durch materielle Zwänge und symbolische Formen der Hegemonieausübung genossenschaftliches Wirtschaften systematisch erschwert. Um darüber Aufschluss zu erhalten, wie Genossenschaften dennoch erfolgreich und sozialreformerisch wirksam sein können, werden verschiedene förderliche Rahmenbedingungen identifiziert: Solidarische Bündnisse der Genossenschaften untereinander und mit anderen Akteuren gelten, wie bereits erwähnt, als die wichtigsten, daneben sollte versucht werden, „Gegen-Institutionen“ aufzubauen (Baldacchino 1990b, S. 470), die

- Produktivgenossenschaften materiell und mit Expertise unterstützen können,

- dazu beitragen, Gesellschaftsmitglieder im Sinne dieser alternativen Logik zu sozialisieren und mit entsprechenden Fähigkeiten und Erfahrungen demokratischer Partizipation auszustatten und

- genossenschaftliche Werte innerhalb der Gesellschaft verbreiten, um Solidarität zwischen aktuellen und potenziellen Genossenschaftsmitgliedern herzustellen.

Daneben müsse es gelingen, gesellschaftlich dominanten Denkformen und Genossenschaftsideen eigene Alternativen im öffentlichen, politischen und akademischen Diskurs gegenüberzustellen und die wissenschaftliche Auseinandersetzung mit Genossenschaften aus einer kritischen Perspektive zu stärken.

Ansatzpunkte für Letzteres werden in diesen Debatten in einer Analyse von Produktivgenossenschaften mit den Werkzeugen der Labour Process Theory (Egan 1990; Davis/

8 „Seit 1851 arbeitete Marx an den von Ernest Jones heraus gegebenen Chartistenzeitungen "Notes to the People" und „The People's Paper" mit. Eine Vielzahl ökonomischer Artikel, die unter Ernest Jones' Namen erschienen, sind unter direkter Mitarbeit von Marx zustande gekommen" (Krätke 2003 , S. 22). 
Parker 2007) gesehen, die als wichtiger Ansatz kritischer Arbeitsforschung im anglo-amerikanischen Raum Arbeitsprozesse aus marxistischer und post-strukturalistischer Perspektive betrachtet (Thompson/Smith 2010). Hier schließt sich der Kreis, denn auch diese Anregung wurde bisher in der anglo-amerikanischen Forschung nicht aufgegriffen. Formen der innerbetrieblichen Kontrolle, der Sicherung von Kooperation und Kreativität der Arbeitenden, formelle und informelle Hierarchien, Prozesse der Identitätsbildung und -sicherung in der Arbeit und widerständige Praktiken der Genossenschaftsmitglieder würden hier ins Zentrum gerückt.

\section{Offene Fragen und Ausblick}

Diese knappe Skizze der theoretischen Diskussion belegt, dass im Bereich der solidarischen Ökonomie bzw. der sozialreformerischen Genossenschaften kritisch geforscht wird, beispielsweise aus marxistischer Perspektive. Dieser, wie auch aktuelle Diskurse anderer Forschungstraditionen könnten sicherlich noch intensiver erschlossen werden. Gleiches gilt für die Diskussionen im Kontext der alternativen Betriebe aus den 1970er und 1980er Jahren sowie der Betriebsübernahmen durch Belegschaften. Nicht nur verspricht dies Erkenntnisse über einen randständigen, aber spannenden Bereich potenziell emanzipatorischer Gestaltung von Arbeit und Wirtschaft, sondern gleichzeitig auch eine Herausforderung und Bereicherung der arbeits- und organisationssoziologischen Forschung. Weitere zentrale und grundlegende Aufgaben zukünftiger soziologischer Forschung im Bereich (sozialreformerischer) Genossenschaften seien abschließend skizziert.

(1) Aus meiner Perspektive scheint es interessant, die aktuell existierenden Projekte wahrzunehmen und akademisch und politisch zu diskutieren besonders an Debatten um Wirtschaftsdemokratie könnte hierbei angeknüpft werden.

(2) Diejenigen Bereiche der Wirtschaft wären vertieft zu analysieren, in denen produktivgenossenschaftliches Wirtschaften besondere Chancen bietet und Erfolg versprechend sein könnte. Personenbezogene soziale Dienstleistungen kommen in dieser Hinsicht strukturell (arbeitsintensiv, ortsgebunden, expandierend) als mögliches Wachstumsfeld infrage.

(3) Freilich müsste sich eine solche Forschung die Grundlagen genossenschaftlichen Wirtschaftens erneut aneignen und mit angemessenen wissenschaftlichen Begriffen empirische Studien durchführen, um die Akteurinnen und Akteure, die Organisation der Arbeit, die spezifischen Erfahrungen und die Lernprozesse produktivgenossenschaftlichen Wirtschaftens analysieren zu können und ihre Aneignung und Übertragbarkeit zu untersuchen.

(4) Sie könnte weiterhin davon profitieren, historisch-vergleichend sowohl die Alternativbetriebe als auch die gewerkschaftliche Gemeinwirtschaft erneut in den Blick zu nehmen. Daran anschließend ließen sich kritische theoretische Überlegungen zu den Problemen, Potenzialen und Rahmenbedingungen genossenschaftlichen Wirtschaftens aufnehmen, prüfen und weiterentwickeln, die betriebliche Autonomie und gesamtgesellschaftliche Strukturen und Prozesse einbeziehen.
(5) Die genossenschaftliche Form der Demokratisierung der Wirtschaft wäre auch unter dem Aspekt der Geschlechterdemokratie zu untersuchen. Die Reaktivierung der Forschung zu Genossenschaften sollte konsequent geschlechtersensibel erfolgen und die Erkenntnisse feministischer Arbeitsforschung integrieren, um den egalitären Anspruch der Genossenschaften auch auf die Geschlechterfrage zu beziehen und ihm in der Forschungsperspektive gerecht zu werden.

Theoretische und empirische Forschungsergebnisse zu diesen Punkten können einerseits von den Akteurinnen und Akteuren sozialreformerischer Genossenschaften und der solidarischen Ökonomie aufgegriffen und angeeignet werden sowie andererseits dazu beitragen, politische und akademische Perspektiven zu erweitern: Das gilt für die (kritische) Arbeitssoziologie genauso wie für die Debatten um Wirtschaftsdemokratie, für die Bestimmung gewerkschaftlicher Positionen gegenüber Genossenschaften wie für konzeptionelle und utopische Überlegungen in Richtung eines progressiven politischen Projektes.

\section{LITERATUR}

Altvater, E./Sekler, N. (Hrsg.) (2006): Solidarische Ökonomie. Reader des Wissenschaftlichen Beirats von Attac, Hamburg

Atzeni, M. /Ghigliani, P. (2007): Labour process and decision-making in factories under workers' self-management: empirical evidence from Argentina, in: Work, employment and society 21 (4), S. 653-671

Baldacchino, G. (1990a): Worker cooperatives with particular reference to Malta. An educationist's theory and practice, Den Haag

Baldacchino, G. (1990b): A War of Position: Ideas on a Strategy for Worker Cooperative Development, in: Economic and Industrial Democracy 11 (4), S. $463-482$

Beerhorst, J. (2009): Vergesellschaftung! Welche Vergesellschaftung? Über Finanzmarktkapitalismus, Krise und Demokratisierung der Wirtschaft, in: Kritische Justiz 42 (2), S. 148-166

Berger, D. (2008): Das Genossenschaftswesen heute - eine Erfolgsgeschichte, in: Förderverein Hermann Schulze-Delitzsch (Hrsg.): Hermann Schulze-Delitzsch, Weg - Werk - Wirkung , Wiesbaden, S. 412-421

Bernardi, J. (2009): Solidarische Ökonomie, Selbstverwaltung und Demokratie in Brasilien und Deutschland, Kassel

Bierbaum, H. (2008): Renaissance der Genossenschaften? Alternative zu finanzmarktgetriebener Unternehmenspolitik, in: Forum Wissenschaft 25 (3), http://www.bdwi.de/forum/archiv/archiv/1663145.html (letzter Zugriff: 4.1.2010) Bierbaum, H. (2011): Renaissance der Belegschaftsbetriebe? Zur Diskussion in den Gewerkschaften, in: Luxemburg. Gesellschaftsanalyse und linke Praxis 3 (3), S. 6-12

Bierbaum, H./Riege, M. (1989): Selbsthilfe, Genossenschaften, Vergesellschaftung. Der Beitrag von Selbsthilfe und neuen Genossenschaften für gesellschaftliche Reformpolitik, Hamburg

Davis, P./Parker, M. (2007): Cooperatives, Labor, and the State:The English Labor Economists Revisited, in: Review of Radical Political Economics 39 (4), S. $523-542$

Dellheim, J. (2005): Genossenschaften bzw. genossenschaftliche Unternehmen und Organisationen - Herausforderung und Thema für linke Politik in Deutschland und EU-Europa, http://www.rosalux.de/cms/fileadmin/rls_ uploads/pdfs/Europa-Studie.pdf (letzter Zugriff: 7.1.2010)

Dellheim, J. (2008): Solidarische Ökonomie - ein Thema für sozialistische Politik, rls Standpunkte (28), Berlin, http://www.rosalux.de/fileadmin/rls_uploads/ pdfs/Standpunkte_0828.pdf (letzter Zugriff: 24.11.2009)

Demirovic, A. (2007): Demokratie in der Wirtschaft. Positionen - Probleme Perspektiven, Münster 
Demirovic, A. (2011): „Wir können das besser“. Belegschaftseigentum, Demokratie und Transformation, in: Luxemburg. Gesellschaftsanalyse und linke Praxis 3 (3), S. 22-31

Egan, D. (1990): Toward a Marxist Theory of Labor-Managed Firms: Breaking the Degeneration Thesis, in: Review of Radical Political Economics 22 (4),

S. $67-86$

Elsen, S. (2007): Die Ökonomie des Gemeinwesens. Sozialpolitik und soziale Arbeit im Kontext von gesellschaftlicher Wertschöpfung und -verteilung, Weinheim

Flieger, B. (Hrsg.) (1984): Produktivgenossenschaften. Oder der Hindernislauf zur Selbstverwaltung. Theorie, Erfahrungen und Gründungshilfen zu einer demokratischen Unternehmensform, München

Flieger, B. (1996): Produktivgenossenschaft als fortschrittsfähige Organisation. Theorie, Fallstudie, Handlungshilfen, Marburg

Flieger, B. (Hrsg.) (2003): Sozialgenossenschaften. Wege zu mehr Beschäftigung, bürgerschaftlichem Engagement und Arbeitsformen der Zukunft, NeuUIm

Flieger, B. (2006): Genossenschaften in Deutschland -Teil der Solidarischen Ökonomie, in: Altvater, E./Sekler, N., a.a.O., S. 47-61

Flieger, B. (2007a): Selbsthilfegenossenschaften. Die Wir-eG: Arbeitsplätze durch unterstützte Gruppenselbsthilfe, in: Müller-Plantenberg, a.a.O.,

S. $143-149$

Flieger, B. (2007b): Das novellierte Genossenschaftsgesetz als Chance für die Sozialwirtschaft, in: Müller-Plantenberg, C., a.a.O., S. 265-273

Giegold, S./Embshoff, D. (Hrsg.) (2008): Solidarische Ökonomie im globalisierten Kapitalismus, Hamburg

Giegold, S./Embshoff, D. (2008): Solidarische Ökonomie im globalisierten Kapitalismus, in: Giegold, S./Embshoff, D. (Hrsg.), a.a.O., S. 11-24

Grosskopf, W./Münkner, H.-H./Ringle, G. (2010): Our Co-op. Idea - Mission Achievements, Neu-UIm

Habermann, F. (2009): Halbinseln gegen den Strom. Anders leben und wirtschaften im Alltag, Königstein/Taunus

Heider, F./Hock, B./Seitz, H.-W. (1997): Kontinuität oder Transformation? Zur Entwicklung selbstverwalteter Betriebe. Eine empirische Studie, Gießen Jones, E. (1979): A letter to the Advocates of the Cooperative Principle, and to the Members of the Cooperative Societies, Marx and Engels Collected Works, Vol. 11, London

Jossa, B. (2005): Marx, Marxism and the cooperative movement, in: Cambridge Journal of Economics 29 (1), S. 3-18

Jossa, B. (2009a): Gramsci and the Labor-Managed Firm, in: Review of Radical Political Economics 41 (1), S. 5-22

Jossa, B. (2009b): Gramsci, the economic theory of cooperatives and the transition to a socialist economy, http://www.sie.univpm.it/incontri/rsa49/Libere/ Lavori/Jossa.pdf (letzter Zugriff: 14.12.2009)

Klemisch, H./Flieger, B. (2007): Genossenschaften und ihre Potenziale für Innovation, Partizipation und Beschäftigung. Der Beitrag von Genossenschaften zur sozialen Verantwortung von Unternehmen, http://www.boeckler.de/pdf fof/S-2006-857-2-1.pdf (letzter Zugriff: 25.04.2012)

Klemisch, H./Sack, K./Ehrsam, C. (2010): Betriebsübernahme durch Belegschaften - Eine aktuelle Bestandsaufnahme, http://www.boeckler.de/pdf fof/S-2009-303-1-1.pdf (letzter Zugriff 4.11.2011)

Kramer, J. W. (2008): Produktivgenossenschaften - Utopische Idee oder realistische Perspektive, Wismarer Diskussionspapiere (12), Wismar

Krätke, M. R. (2003): Wirtschaftsdemokratie und Marktsozialismus, http://www. praxisphilosophie.de/kraetkewd.pdf (letzter Zugriff: 9.12.2009)

Krätke, M. R. (2008): Über Wirtschaftsdemokratie, in: transform! 1 (2),

S. $93-103$

Luxemburg, R. (1899): Sozialreform oder Revolution?, http://www.marxists. org/deutsch/archiv/luxemburg/1899/sozrefrev/kap2-2.htm (letzter Zugriff 20.12.2009)
Martens, H. (2010): Neue Wirtschaftsdemokratie. Anknüpfungspunkte im Zeichen der Krise von Ökonomie, Ökologie und Politik, Hamburg

Marx, K. (1973[1866 ]): On the First International. Instructions for the Delegates of the Provisional General Council, New York

Mersmann, A./Novy, K. (1991): Gewerkschaften, Genossenschaften, Gemeinwirtschaft. Hat eine Ökonomie der Solidarität eine Chance?, Köln

Müller-Plantenberg, C. (Hrsg.) (2005): Solidarische Ökonomie in Brasilien und Deutschland. Wege zur konkreten Utopie, Kassel

Müller-Plantenberg, C. (Hrsg.) (2007): Solidarische Ökonomie in Europa. Betriebe und regionale Entwicklung, Kassel

Müller-Plantenberg, C. (2007): Solidarische Ökonomie und Regionale Entwicklung, in: Müller-Plantenberg, C. (Hrsg.), a.a.O., S. 55-67

Neumann, A. (2008): Kleine geile Firmen. Alternativprojekte zwischen Revolte und Management, Hamburg

Notz, G. (1991): Gibt es ein richtiges Leben im falschen? - Frauen in der Selbstverwaltungswirtschaft, in: Notz, G. (Hrsg.): Selbstverwaltung in der Wirtschaft. Alte Illusion oder neue Hoffnung?, Köln, S. 188-215

Notz, G. (2004): Ein Fenster in eine herrschaftsfreie Welt. Das Demokratiepotenzial von Alternativökonomie, selbstverwalteten Betrieben und kommunitären Lebens- und Arbeitsformen - ein Positionsbeitrag, in: Weber, W. G. (Hrsg.) Wirtschaft, Demokratie und soziale Verantwortung. Kontinuitäten und Brüche, Göttingen, S. 265-280

Notz, G. (2011): Theorien alternativen Wirtschaftens. Fenster in eine andere Welt, Stuttgart

Plogstedt, S. (2006): Frauenbetriebe. Vom Kollektiv zur Einzelunternehmerin Königstein/Taunus

Priller, E./Zimmer, A. (2006): Dritter Sektor: Arbeit als Engagement, in: Aus Politik und Zeitgeschichte 56 (12), S. 17-24

Projektgruppe Solidarische Ökonomie der Universität Kassel (2008): Atlas der Solidarischen Ökonomie in Nordhessen. Strategie für eine nachhaltige Zukunft, Kassel

Schlosser, I./Zeuner, B. (2006): Gewerkschaften, Genossenschaften und Solidarische Ökonomie, in: Altvater, E./Sekler, N. (Hrsg.), a.a.O, S. 32-39 Thompson, P./Smith, C. (2010): Working life. Renewing labour process analysis, Houndmills

Uhl, A. (2011): Der Kongress, der viele ist. Theoretische Erörterung von (anti-) repräsentativen Praxen am Beispiel des Wiener Solidarökonomie Kongresses 2009, in: Grundrisse (39), http://www.grundrisse.net/grundrisse39/kongress der_viele_ist.htm (letzter Zugriff: 4.11.2011)

Vilmar, F. (1999): Wirtschaftsdemokratie - Zielbegriff einer alternativen Wirtschaftspolitik. Kritische Bilanz und Aktualität nach 40 Jahren, http://www. memo.uni-bremen.de/docs/m3206.pdf (letzter Zugriff: 9.12.2009)

Voß, E. (2010): Wegweiser Solidarische Ökonomie. Anders wirtschaften ist möglich!, Neu-Ulm

\section{AUTOR}

STEFAN KERBER-CLASEN, Soziologe M.A., ist Mitarbeiter am Institut für Sozialforschung und Sozialwirtschaft (iso), Saarbrücken. Arbeitsschwerpunkte: Arbeitssoziologie, Formen alternativen Wirtschaftens.

stefan.kerber@gmx.net 